\title{
Epidemiological network analysis in HIV-1 B infected patients diagnosed in Italy between 2000 and 2008
}

\author{
Annapaola Callegaro ${ }^{a}$, Valentina Svicher ${ }^{b}$, Claudia Alteri $^{\mathrm{b}}$, Alessandra Lo Presti ${ }^{\mathrm{c}}$, \\ Daniela Valenti ${ }^{\mathrm{d}}$, Antonio Goglio ${ }^{\mathrm{a}}$, Marco Salemi ${ }^{\mathrm{e}}$, Eleonora Cella ${ }^{\mathrm{c}}$, Carlo Federico Perno ${ }^{\mathrm{b}}$, \\ Massimo Ciccozzi ${ }^{\mathrm{c}, *}$, Franco Maggiolo ${ }^{\mathrm{d}}$ \\ ${ }^{a}$ Microbiology and Virology, Ospedali Riuniti di Bergamo, Italy \\ ${ }^{\mathrm{b}}$ Department of Experimental Medicine, University of Rome "Tor Vergata", Italy \\ ${ }^{\mathrm{c}}$ Department of Infectious Diseases Istituto Superiore di Sanità, Italy \\ ${ }^{\mathrm{d}}$ Division of Infectious Diseases, Ospedali Riuniti di Bergamo, Italy \\ e Department of Pathology Immunology and Laboratory Medicine, University of Florida, Gainesville, FL, United States
}

\section{A R T I C L E I N F O}

\section{Article history:}

Received 13 January 2011

Accepted 23 January 2011

Available online 1 February 2011

\section{Keywords:}

Epidemiological network

HIV-1 subtype B epidemic

Phylogenetic analysis

Time-scaled phylogenies

Transmitted drug-resistance (TDR)

\begin{abstract}
A B S T R A C T
This study, through a phylogenetic analysis, is aimed to identify potential epidemiological networks and sequence interrelationships between acute/early and chronic infections in both drug-naïve and drugexperienced individuals within a local, well-defined setting and to investigate the population dynamics of transmitted resistance and the potential contribution of untreated patients to the spread of antiretroviral resistance. A total of 884 HIV-1 B subtype pol gene sequences from 306 drug-naïve (40 recently and 266 chronically infected) and 578 drug-treated HIV-1 infected patients were collected through routine drug-resistance testing between 2000 and 2008 in a single center (Division of Infectious Disease, Bergamo, Northern Italy). Bayesian phylogenetic tree was reconstructed and transmission clusters were recognized using a posterior probability as statistical support of each cluster. Differences among clustered and non-clustered drug-resistance mutations were assessed by Fisher's exact test.

In our cohort we identified five clusters including $\geq 6$ sequences with the root posterior probability of $100 \%$. Dated phylogenies reconstructed through Bayesian Markov chain Monte Carlo model was possible for only two main clade ( $\geq 10$ sequences) originated between 1990 and 2002.

Among the 306 drug-naïve individuals, $12 \%$ carried a viral strain with at least 1 major mutation associated with transmitted drug resistance and $36 \%$ of these strains were involved in significant clusters.

We report for the first time that many (34\%) of HIV-1 subtype B transmission clusters indentified in Italy were only composed by drug-naïve individuals and that the $14 \%$ of transmitted drug resistance was linked to transmission clusters composed only of newly diagnosed individuals. The phylogenetic analysis was performed on a large cohort of drug-naïve recently/chronically infected individuals where drug-experienced patients represent almost all infected individuals in a restricted geographical area.

Our findings highlight the role of newly diagnosed individuals, not yet exposed to antiretroviral drugs, in the transmission of drug-resistant HIV-1 strains, providing new insights for the planning and management of treatment programs in developing countries.
\end{abstract}

(c) 2011 Elsevier B.V. All rights reserved.

\section{Introduction}

At the end of the year 2008, in Italy, 60,346 persons were cumulatively diagnosed as affected by AIDS (Suligoi et al., 2009).

\footnotetext{
* Corresponding author at: Department of Infectious Diseases, Istituto Superiore di Sanità, Viale Regina Elena 299, Roma, Italy. Tel.: +39 06 49903187; fax: +3906 49902610.

E-mail address: ciccozzi@iss.it (M. Ciccozzi).
}

Overall, it is estimated that 140,000/150,000 HIV-positive people live within the country. At the beginning of the epidemics the most common route of transmission for HIV was needle sharing among intravenous drug users (IDUs) (Castelnuovo et al., 2003) that counted for more than $50 \%$ of cases. In more recent years heterosexual contacts have become the leading cause of infection (44.7\%), while homosexual contacts have maintained a quite constant incidence varying from $15.5 \%$ in 1997 to $20.8 \%$ in 2008 (Suligoi et al., 2009). Cases are not evenly distributed throughout the nation and the region of Lombardia in the Northern part of the 
country contributed with 21,506 AIDS cases to the general picture. Within the region, the highly populated and industrialized province of Bergamo shows one of the highest national incidence rates of new cases: 3.6 cases for 100,000 inhabitants (Suligoi et al., 2009).

In Italy, the human immunodeficiency virus type 1 (HIV-1) epidemic is still mainly sustained by subtype B genetic form, although other and novel subtypes and circulating recombinant forms (CRFs) have been reported (Baldanti et al., 2008; Balotta et al., 2001; Buonaguro et al., 2008; Ciccozzi et al., 2007).

After the introduction of highly active antiretroviral therapy (HAART) in the mid-1990s, drug-resistant HIV-1 strains to every class of drugs have been documented (Costagliola et al., 2007; Pillay et al., 2000; Tozzi et al., 2006; Violin et al., 2004a).

To date, there is a growing literature about the rate of transmission of HIV-1 drug-resistant virus. In the United States and in Europe, where there is a wide access to highly active antiretroviral therapy (HAART), the prevalence of HIV-1 drugresistant strains ranges between $3.3 \%$ and $15.0 \%$ in recently infected patients and between $6.1 \%$ and $12.5 \%$ in chronically infected ones (Costagliola et al., 2007; The SPREAD Programme, 2008; UK Collaborative Group on HIV Drug Resistance et al., 2007; Violin et al., 2004a,b; Wensing et al., 2005; Yerly et al., 2001, 2007, 2009). This prevalence is in line with than reported in Italy by several epidemiological reports (prevalence of drug resistance strains in drug naïve patients ranging from 6 to 15\%)(Alteri et al., 2009; Balotta et al., 2001; Buonaguro et al., 2008; Lapadula et al., 2008).

Very recently it was clear that the transmission of drugresistant HIV-1 viruses does not only reflect infection from drugexperienced patients, but also the circulation of resistant strains between drug-naïve individuals (Hue et al., 2009).

With this study, using a phylogenetic approach, we attempted to identify potential epidemiological networks and sequence interrelationships between acute/early and chronic infections in both drug-naïve and drug-experienced individuals within a local, well-defined setting.

We also sought to investigate the population dynamics of transmitted resistance and the potential contribution of untreated patients to the spread of antiretroviral resistance.

The demonstration of those epidemiological events can provide new tools in prevention strategy as well as in management of treatment programs in a local, specific and well-characterized territory.

\section{Materials and methods}

\subsection{Patients and dataset}

A total of 884 HIV-1 B subtype pol gene sequences (containing the full-length protease [PR] and the first 344 reverse transcriptase [RT]) codons from 306 drug-naïve (40 recently [PHI or $<6$ months after seroconversion], and 266 chronically infected) and 578 drugtreated HIV-1 infected patients were analyzed. A recent infection was defined as follows: (1) clinic signs of primary HIV infection (HIV Rna level $>10,000$ copies/ml and negative or indeterminate HIV-antibody test) at the time of diagnosis; (2) a documented negative result of a HIV screening test within 6 months before HIV diagnosis; (3) laboratory evidence of seroconversion $<6$ months. All sequences were collected through routine drug-resistance testing between 2000 and 2008 in a single center (Division of Infectious Disease, Bergamo) located in the Northern part of Italy. For drug-naïve patients, the sample closest to the date of HIV-1 diagnosis was selected for this analysis, while, for drug-experienced individuals, the last (in order of time) was used.

The sequences were coded for anonymity, but information on geographical region, self-reported route of transmission, and date of sampling was retained.

\subsection{HIV-1 genotyping}

Viral RNA was extracted from the patients' plasma, reverse transcripted, amplified and sequenced using The Viroseq HIV-1 Genotyping System (Celera Diagnostics, CA, USA) according to the manufacturer's instructions.

Capillary electrophoresis was undertaken on the ABI-Prism3130 Genetic Analyzer. Resistance mutation analysis was performed using the ViroSeq System Software.

Sequence data were submitted to Stanford Resistance Database tool (http://hivdb.stanford.edu) to obtain an estimate of the level of susceptibility to antiretrovirals and the identification of the HIV-1 subtype. In addition all sequences were analyzed using the REGA HIV1 subtyping tool [http://dbpartners.stanford.edu/RegaSubtyping].

\subsection{Phylogenetic analysis}

To avoid the influence of convergent evolution at antiretroviral drug-resistance mutations on the phylogenetic analysis, a codonstripped dataset from which 44 codons associated with major resistance in PR (positions 30, 32, 33, 46, 47, 48, 50, 54, 58, 74, 76, $82,84,88,90$ ) and RT (positions 41, 62, 65, 67, 70, 74, 75, 77, 90, 98, $100,101,103,106,108,115,116,138,151,184,179,181,188,190$, $210,215,219,225,230)$ was analyzed.

The first 1302 nucleotides of pol gene from 884 HIV-1 B subtype infected patients were analyzed. All the sequences were aligned, removing gaps and cutting to identical sequence lengths using ClustalX software (Thompson et al., 1994). The manual editing was performed using Bioedit software (Hall, 1999).

Phylogenetic approach was used to establish clustered transmission and sequence interrelationships between acute/early and chronic infections in both treated and not treated patients.

Multiple alignments spanning subtype specific HIV-1 pol sequences were analyzed by Bayesian phylogenetic methods.

Bayesian phylogenetic tree was reconstructed by $\mathrm{Mr}$ Bayes (Huelsenbeck and Ronquist, 2001) using a GTR + I + G model of nucleotide substitution and a proportion of invariant sites and gamma distribuited rates among sites. MCMC search was conducted for $5 \times 10^{6}$ generations, with tree sampled every 100 th generation and a burn in of $50 \%$. Statistical support for specific clades was obtained by calculating the posterior probability of each monophyletic clade and a posterior consensus tree was generated after a burn in of $50 \%$. Transmission clusters were recognized on the basis of the same tree and a posterior probability was used as statistical support of each cluster. Cluster group size was determined using nodes with a posterior probability of 1 .

A second Bayesian phylogenetic tree including pol gene from our 884 HIV-1 B subtype infected patients plus pol gene of foreign HIV-1 B reference sequences downloaded from HIV Los Alamos (http://www.hiv.lanl.gov/content/index) was reconstructed by $\mathrm{Mr}$ Bayes (Huelsenbeck and Ronquist, 2001) using the same model specified above (Fig. S1), to prove that Italian clusters were not split by foreign sequences.

Inclusion criteria for the sequences downloaded by Los Alamos were:

(1) sequences had already been published in peer-review journals (except for the new sequences described below); (2) there was no uncertainty about the subtype assignment for each sequence and were classified as non recombinants; (3) sequences were not epidemiologically linked by direct donor-recipient transmission; (4) only one sequence per individual could be randomly selected; and (5) city/state of origin and sample date were known and clearly established in the original publication.

Statistical support for specific clades was obtained by calculating the posterior probability of each monophyletic clade. 
Transmission clusters were recognized on the basis of the same tree and a posterior probability was used as statistical support of each cluster. Cluster group size was determined using nodes with a posterior probability of 1 .

Likelihood mapping of 10,000 random quartets of all HIV-1 B sequences was generated using TreePuzzle software (Schmidt et al., 2002). Each dot represents the likelihoods of the three possible unrooted trees for a set of four sequences (quartets) selected randomly from the data set: dots close to the corners or the sides represent, respectively, tree-like, or network-like phylogenetic signal in the data. The central area of the likelihood map, represents star-like signal. The percentage of dots in the central area is given at the basis of each map.

\subsection{Genotypic resistance analysis}

According to International Guidelines, a total of 88 mutations associated with transmitted drug resistance were analyzed: L23I, L24I, D30N, V32I, M46I/L, I47A/V, G48V, I50V/L, F53L, I54A/M/L/V/ T/S, G73A/C/S/T, L76V, V82A/C/L/F/M/T/S, N83D, I84A/C/V, I85V, $\mathrm{N} 88 \mathrm{D} / \mathrm{S}$, and L90M in PR, and M41L, K65R, D67N, T69ins/D, K70R/E, L74V/I, V75A/M/T/S, F77L, L100I, K101E/P, K103N/S, V106A/M, Y115F, F116Y, Q151M, V179F, Y181C/I/V, M184V/I, Y188C/H/L, G190A/E/S, L210W, T215Y/F/C/D/E/I/V, K219E/N/R/Q, P225H, M230L in RT) (4). Polymorphisms at positions already associated with drug resistance were also investigated.

For each tree, the phylogenetic clustering of at least one drugresistant sequence from a drug-naïve patients was considered evidence for the existence of a drug-resistant viral lineage. Differences among clustered and non-clustered drug-resistance mutations were assessed using Fisher's exact tests.

\subsection{Time-scaled phylogenies}

For each cluster group involving $\geq 6$ sequences, the dated trees, evolutionary rates and population growth were co-estimated by using a Bayesian MCMC approach (Beast version 1.4.8. http:// beast.bio.ed.ac.uk) (Drummond and Rambaut, 2007) implementing a GTR + Invariant + Gamma model. A log normal prior to the evolutionary rates was used and rates were estimated taking into account the known sampling time of the sequences.

As coalescent prior, different parametric demographic models (a constant population size, and exponential and logistic growth) and a non-parametric Bayesian skyline plot (BSP) were compared under strict and relaxed clock conditions, and the best models were selected by means of a Bayes factor (BF, using marginal likelihoods) implemented in Beast (Suchard et al., 2001). In accordance with Kass and Raftery (Kass and Raftery, 1995), the strength of the evidence against $\mathrm{HO}$ was evaluated as follows: $2 \ln \mathrm{BF}<2$ no evidence; 2-6 weak evidence; 6-10 strong evidence; $>10$ very strong evidence. A negative value indicates evidence in favour of $\mathrm{H} 0$. Only values of $\geq 6$ were considered significant.

Chains were conducted for $100 \times 10^{6}$ generations, and sampled every 10,000 steps for clusters A-D, whereas for cluster $E$ the analysis was conducted for $50 \times 10^{6}$ generations, and sampled every 5000 steps. Convergence was assessed on the basis of the effective sampling size (ESS) after a $50 \%$ burn-in using Tracer software version 1.4 (http://tree.bio.ed.ac.uk/software/tracer). Only parameter estimates with ESS's of $>200$ were accepted. Uncertainty in the estimates was indicated by $95 \%$ highest posterior density (95\% HPD) intervals.

The maximum clade credibility tree has been produced by the original version of TreeAnnotator (Drummond and Rambaut, 2007) as the tree in the posterior sample that has the maximum sum of posterior clade probabilities.
Table 1

Demographic, clinical and virological characteristics of the cohort.

\begin{tabular}{llll}
\hline & Overall & Drug-naïve & Drug-experienced \\
\hline Number of patients & 884 & 306 & 578 \\
Male, $N(\%)$ & $681(77)$ & $258(84)$ & $423(73)$ \\
Italian, $N(\%)$ & $845(95)$ & $276(90)$ & $569(98)$ \\
HIV exposure, $N(\%)$ & & & \\
MSM & $156(18)$ & $93(31)$ & $63(11)$ \\
Heterosexual & $330(38)$ & $146(50)$ & $184(32)$ \\
IDUs & $382(44)$ & $56(19)$ & $326(57)$ \\
Unknown, $N$ & 16 & 11 & 5 \\
Age (years), & $38(34-43)$ & $40(33-46)$ & $38(34-42)$ \\
Median $(I Q R)$ & & & \\
Viral Load, & $4.3(3.7-4.9)$ & $4.8(4.5-5.5)$ & $4(3.5-4.5)$ \\
Median $(I Q R)$ & & & \\
Log Copies/ml & & $317(124-471)$ & $353(210-507)$ \\
CD4 cell/mm ${ }^{3}$ & $344(182-499)$ & & \\
\hline
\end{tabular}

\section{Results}

\subsection{Patient's characteristics}

This study includes 884 HIV-1 B subtypes infected patients followed in a single clinical center in North Italy between January 2000 and July 2008. Among them, 40 (4.5\%) were classified having a recent infection (PHI $<6$ months after seroconversion), 266 (30.1\%) as chronically infected patients and 578 (65.4\%) as drugexperienced HIV-1 infected patients. The main exposure groups were all represented: men having sex with men (MSM; $n=156$ ), heterosexual transmission (Heterosexual; $n=330$ ), injection drug users (IDUs; $n=382$ ), and other exposure group $(n=16)$. All the other epidemiological and clinical data of our cohort are summarized in Table 1.

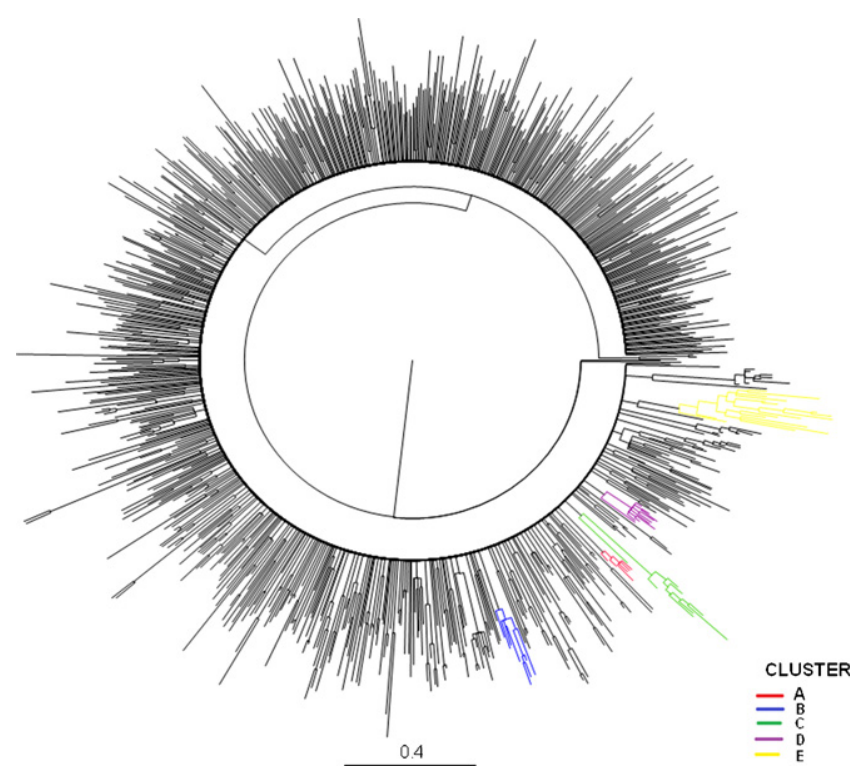

Fig. 1. Bayesian phylogenetic tree of the 884 HIV-1 B subtype pol gene sequences molecular clock, after exclusion of 44 codon positions associated with drug resistance. Branch lengths are expressed as the number of nucleotide substitutions per site. The five clusters in the trees are highlighted with different colours reported in the legend. For each cluster the statistical support $(p<0.001$ zero length branch test and the posterior probability [100\%]) has been computed. (For interpretation of the references to colour in this figure legend, the reader is referred to the web version of this article.) 


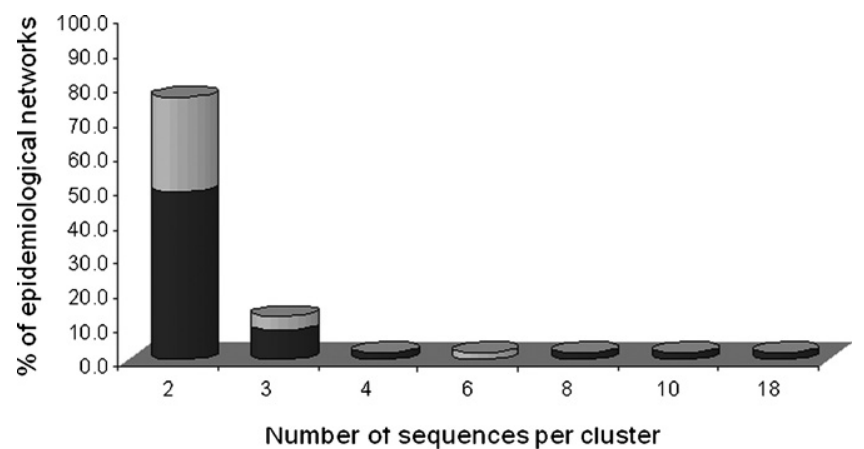

Naïve-Naive

Naïve-Treated

Fig. 2. The distribution of drug-naïve and drug-experienced patients in the 47 clusters. Overall, $76.6 \%$ of the chains have 2 persons/cluster, whereas the remaining individuals are in clusters having $>2$ persons/cluster. Single clustering of drugexperienced patients and non-clustered drug-naïve patients is also depicted.

\subsection{Phylogenetic analysis}

The phylogenetic tree involving all the 884 HIV-1 B sequences is shown in Fig. 1. In this approach, we define clusters as clades identified with a posterior probability of 1 .

Tree topology revealed that 89 (29.1\%) out of 306 drug-naïve patients grouped into 47 significant clusters. Thirty-six [76.6\%] involved 2 patients, while the remaining 11 involved from 3 to 18 patients. In addition, 16 (34.0\%) out of 47 significant clusters involved drug-naïve patients only, while the remaining 31 (66.0\%) involved both naïve and HAART treated patients (Fig. 2).
Among the 40 recent infections, 7 (16.7\%) and 3 (7.5\%) were significantly clustered with untreated and treated chronically infected patients, respectively.

The second Bayesian phylogenetic tree shows that no foreign reference HIV-1 B subtype pol sequences are intermixed in our cluster indicating that our clusters represent local transmission networks (Fig. S1).

The phylogenetic structure of HIV epidemic was also investigated by likelihood mapping analysis (Fig. S1). The evaluation of 10,000 random quartets with the likelihood-mapping method showed a more than $90 \%$ of the randomly chosen quartets from the alignment were distributed in the corner of the likelihood map, which represent tree-like signal.

\subsection{Identification of antiretroviral drug-resistant HIV-1 lineages in drug naive patients}

Among the 306 drug-naïve HIV-1 B subtype infected patients, $36(11.8 \%)$ carried a viral strains with at least 1 major mutation associated with transmitted drug resistance (Table 2) (Bennett et al., 2009). The overall prevalence of mutations conferring resistance to NRTIs, NNRTIs, and PIs was 5.9, 5.6, and $1.0 \%$, respectively. Among these 36 patients, only one patient had a recent infection. This datum may suggest a decreasing trend of drug resistance in recent infections compared to chronic infections (2.4\% in recent infections versus $13.3 \%$ in chronic infections, $p=0.04)$, that has been described also in other Italian reports (Alteri et al., 2009).

Of the 36 drug-resistant strains, 23 (63.9\%) remained unclustered, while $13(36.1 \%)$ were involved in 9 significant clusters. Of this latter group, $8(61.5 \%)$ strains clustered with HAART treated patients, while 5 (38.5\%) with drug-naïve patients.

Table 2

Prevalence of drug-resistance mutations in clustered and no-clustered drug-naïve resistant strains.

\begin{tabular}{|c|c|c|c|c|c|c|}
\hline & $\begin{array}{l}\text { Naïve } \\
N=306\end{array}$ & $\begin{array}{l}\text { Recently infected } \\
N=40\end{array}$ & $\begin{array}{l}\text { Chronically infected } \\
N=266\end{array}$ & Clustered $^{\mathrm{b}}$ & Cluster naïve-treated ${ }^{\mathrm{c}}$ & $\begin{array}{l}\text { Cluster } \\
\text { naïve-naïve }\end{array}$ \\
\hline At least one drug resistance mutation ${ }^{a}$ & $36(11.8)$ & $1(2.5)$ & $35(21.4)$ & $13(36.1)$ & $8(61.5)$ & $5(38.5)$ \\
\hline At least one NRTI resistance & $18(5.89)$ & $0(0.0)$ & $18(6.7)$ & $7(38.9)$ & $4(57.2)$ & $3(42.8)$ \\
\hline At least one TAM & $17(5.6)$ & $0(0.0)$ & $17(6.3)$ & $4(23.5)$ & $2(50.0)$ & $2(50.0)$ \\
\hline M41L & $8(2.6)$ & $0(0.0)$ & $8(3.0)$ & $2(25.0)$ & $2(100.0)$ & $0(0.0)$ \\
\hline D67N & $5(1.6)$ & $0(0.0)$ & $5(1.8)$ & $2(40.0)$ & $0(0.0)$ & $2(100.0)$ \\
\hline T69D & $3(1.0)$ & $0(0.0)$ & $3(1.1)$ & $1(33.3)$ & $0(0.0)$ & $1(100.0)$ \\
\hline K70R & $4(1.3)$ & $0(0.0)$ & $4(1.5)$ & $0(0.0)$ & - & - \\
\hline L74V & $1(0.3)$ & $0(0.0)$ & $1(0.4)$ & $0(0.0)$ & - & - \\
\hline $\mathrm{M} 184 \mathrm{~V} / \mathrm{I}$ & $6(2.0)$ & $0(0.0)$ & $6(2.2)$ & $1(16.7)$ & $1(100.0)$ & $0(0.0)$ \\
\hline L210W & $3(1.0)$ & $0(0.0)$ & $3(1.1)$ & $0(0.0)$ & - & - \\
\hline T215rev & $8(2.6)$ & $0(0.0)$ & $8(3.0)$ & $2(25.0)$ & $1(50.0)$ & $1(50.0)$ \\
\hline $\mathrm{T} 215 \mathrm{~F}$ & $2(0.7)$ & $0(0.0)$ & $2(0.7)$ & $0(0.0)$ & - & - \\
\hline T215Y & $3(1.0)$ & $0(0.0)$ & $3(1.1)$ & $0(0.0)$ & - & - \\
\hline K219Q/E & $7(2.3)$ & $0(0.0)$ & $7(2.6)$ & $2(28.6)$ & $0(0.0)$ & $2(100.0)$ \\
\hline At least one NNRTI resistance & $17(5.6)$ & $1(2.5)$ & $16(6.0)$ & $6(35.3)$ & $4(66.7)$ & $2(33.3)$ \\
\hline K101E & $3(1.0)$ & $0(0.0)$ & $3(1.1)$ & $0(0.0)$ & - & - \\
\hline $\mathrm{K} 103 \mathrm{~N} / \mathrm{S}$ & $11(3.6)$ & $0(0.0)$ & $1(14.1)$ & $3(27.3)$ & $1(33.3)$ & $2(66.7)$ \\
\hline V106A & $1(0.3)$ & $0(0.0)$ & $1(0.4)$ & $0(0.0)$ & - & - \\
\hline V179F & $1(0.3)$ & $0(0.0)$ & $1(0.4)$ & $1(100.0)$ & $1(100.0)$ & $0(0.0)$ \\
\hline Y181C & $2(0.7)$ & $0(0.0)$ & $2(0.7)$ & $1(50.0)$ & $1(100.0)$ & $0(0.0)$ \\
\hline G190A/E/S & $7(2.3)$ & $1(2.5)$ & $6(2.2)$ & $2(28.6)$ & $2(100.0)$ & $0(0.0)$ \\
\hline At least one PI resistance & $3(1.0)$ & $0(0.0)$ & $3(1.1)$ & $0(0.0)$ & - & - \\
\hline D30N & $2(0.7)$ & $0(0.0)$ & $0(0.0)$ & $0(0.0)$ & - & - \\
\hline I54L & $1(0.3)$ & $0(0.0)$ & $1(0.4)$ & $0(0.0)$ & - & - \\
\hline N88D & $2(0.7)$ & $0(0.0)$ & $2(0.7)$ & $0(0.0)$ & - & - \\
\hline L90M & $2(0.7)$ & $0(0.0)$ & $2(0.7)$ & $0(0.0)$ & - & - \\
\hline
\end{tabular}

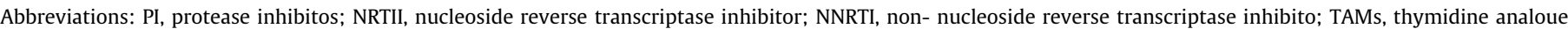
associated mutations.

a Mutations associated with transmitted drug resistance (Bennett et al., 2009) are reported.

b Number (\%) of drug resistance mutations occuring in the overall clusters that have been detected.

c Number (\%) of drug resistance mutations that occurs in clusters involving only drug-naive patients or drug-naive plus HARRT-treated patientsù. 
In particular, the 8 drug-resistant strains clustering with HAART treated patients were involved in 6 significant clusters. These treatment-dependent transmitted drug-resistance mutations consisted of phylogenetically linked mutations shared by drug-treated and drug-naïve patients. Therefore, these clusters of resistant viruses probably reflect transmission from treated to untreated individuals. These clusters are involved in 2 cases of the TAM 1 resistance mutations M41L and T215Y (this last one is present as a recombinant form $\mathrm{T} 215 \mathrm{~S}$ in the drug-naïve patient), the NNRTI resistance mutations G190A + V179F, K103N and Y181C in 3 cases, and finally in one case of the NRTI mutations M41L, M184V and the NNRTI mutation G190E.

Differently, the 5 drug-resistant strains clustering with drugnaïve patients were present in 3 significant clusters. These treatment-independent transmitted drug-resistance mutations were identified as resistance mutations circulating only within phylogenetically linked drug-naïve patients and probably represent drug-resistant HIV-1 populations naturally circulating within untreated individuals. Drug-resistance mutations involved in these clusters were the TAM2 D67N and K219Q, the revertant T215D, and the NNRTI resistance mutation K103N.

Of note, nearly half of NRTI and NNRTI resistance mutations (38.9\% and $35.3 \%$, respectively) were included in epidemiological networks, while PI resistance mutations were never found in clustered transmission.

In addition to clusters involving drug-naïve patients with transmitted drug-resistance, we could also identify 11 clusters, involving as drug-naïve as drug-experienced individuals, where drug-resistance mutations were observed only in drug-experienced patients. All these clusters involved viruses with complex patterns of drug-resistance mutations, which have a negative impact on drug susceptibility and viral replicative capacity.

\subsection{Description of the major lineages}

The 5 clusters ( $\geq 6$ sequences), (posterior probability $=1$ ) were further analyzed to better identify a possible epidemiological network. For each cluster, an exponential model of population growth was significantly favored over a constant and a logistic population size model (data not shown).

Among the 5 clusters analyzed (Fig. 3 and Table 3), 3 (clusters A, $B$ and $E$ ) revealed some degree of internal structure; indeed for each cluster two subclades (Fig. 3) could be identified.

In particular, cluster A included samples from 2006 to 2008 from 6 drug-naïve patients, 5 with chronic and 1 with a recent infection; all patients harbored viruses with no drug-resistance mutations but one of them carried an atypical mutation at a known drug-resistance position L210S (Fig. 3A).

Cluster B included samples enrolled from 2005 to 2008 from 8 drug-naïve patients, all with chronic infection. Again, all patients harbored viruses with no drug-resistance conferring mutations but with an atypical mutation at a known drug-resistance position (PR V82I). In this cluster, the existence of two subclades seems to be closely related with risk factor and nation of origin; indeed, in the first subclade all patients were MSM, with the exception of one patient who declared to be heterosexual, and all patients came from Italy, while in the second subclade all 3 patients were heterosexual and 2 of them came from Morocco (Fig. 3B).

Cluster $\mathrm{E}$ is the most complex lineage identified in our population; it is composed of two subclades and includes 6 drug resistant strains from HAART-treated patients, and 3 resistant strains from drug-naïve patients, observed between 2002 and 2008. The RT mutations M41L, M184V, and G190E were detected in both drug-experienced and drug-naïve patients. In addition, the NNRTI resistance mutation K103N was found in the first subclade, and G190A/E in the second.
This lineage analysis also showed some evidence of reversions to wild type. TAMs, D67N, K70R, L210W, T215Y/F and K219E, the NNRTI mutations K103N and G190A, and the PI resistance mutations D30N and L33F were lost before transmission to drug-naïve individuals; their absence in the earliest sequences of the cluster suggests a long interval between transmission events or a transmission route through individuals not present in this data set (Fig. 3E). Finally, clade $C$ and $D$ included viruses sampled from 8 and 10 subjects enrolled between 2004 and 2008. Both these clusters involved recently infected patients ( 3 in cluster $C$ and 2 in cluster D). Drug-resistance mutations were found only in HAARTtreated patients, while only one drug-naïve patient carried the L210S atypical mutation.

\subsection{Timing of origin of the major lineages}

The time of origin of the resistant lineages was determined using a Bayesian MCMC approach. Each viral sequence in the timescaled phylogenies represents a different patient. For any two sequences, the branches connecting them through their most recent ancestor (MRCA) seems to include at least one possible epidemiological event. The distance between their recent ancestor and the previous node estimates the upper bound of time between epidemiological events. Fig. 3D and $\mathrm{E}$ also shows the variation in internode distances. This representation is scaled by calendar year, from which we can infer the periods over which these clustered transmissions have occurred.

According to these estimates, the two main clade originated between 1990 (95\% highest posterior density, 1983-1993 [cluster E]) and 1997 (95\% highest probability density, 1994-2002 [cluster D]). The time-scaled phylogeny for clusters A-C, was not possible because the short time period and the small sample size.

\section{Discussion}

We report for the first time that many (34\%) of HIV-1 subtype B transmission clusters indentified in Italy were only composed by drug-naïve individuals and that the $14 \%$ of transmitted drug resistance was linked to transmission clusters composed only of newly diagnosed individuals. The phylogenetic analysis was performed on a large cohort of drug-naïve recently/chronically infected individuals and drug-experienced patients represent almost all infected individuals in a restricted geographical area. The phylogenetic structure of the observed epidemics, characterized by high population density and a strict epidemiological link among individuals, indicated a strong signal for a cluster distribution as occurred for the spread of HIV-1 subtype B in Western Europe and North America (Walker et al., 2005).

By analyzing the prevalence of transmitted drug resistance, we found that among the 40 recently infected patients only a single patient carried drug-resistant strains. This result is consistent with the other studies led in Italy and in other European countries, and may suggest a reduction in transmitted drug-resistance (Alteri et al., 2009; The SPREAD Programme, 2008; UK Collaborative Group on HIV Drug Resistance et al., 2007; Yerly et al., 2007). However, we should acknowledge that other European studies reported a stable frequency of transmitted drug resistance in patients at the time of primary HIV-1 infection, while the Swiss cohort reported an increasing trend of transmitted drug resistance, in particular for NNRTI resistance (Yerly et al., 2009). These differences can be explained by the different use of antiretroviral drugs in HIV-1 treated patients in the different settings analyzed. In the attempt to better explain our results, we have performed a further analysis in our cohort of HIV-1 treated patients. This analysis has shown a progressive decrease in the use of NNRTI starting from 2004 , that could be in line with the lower percentage 

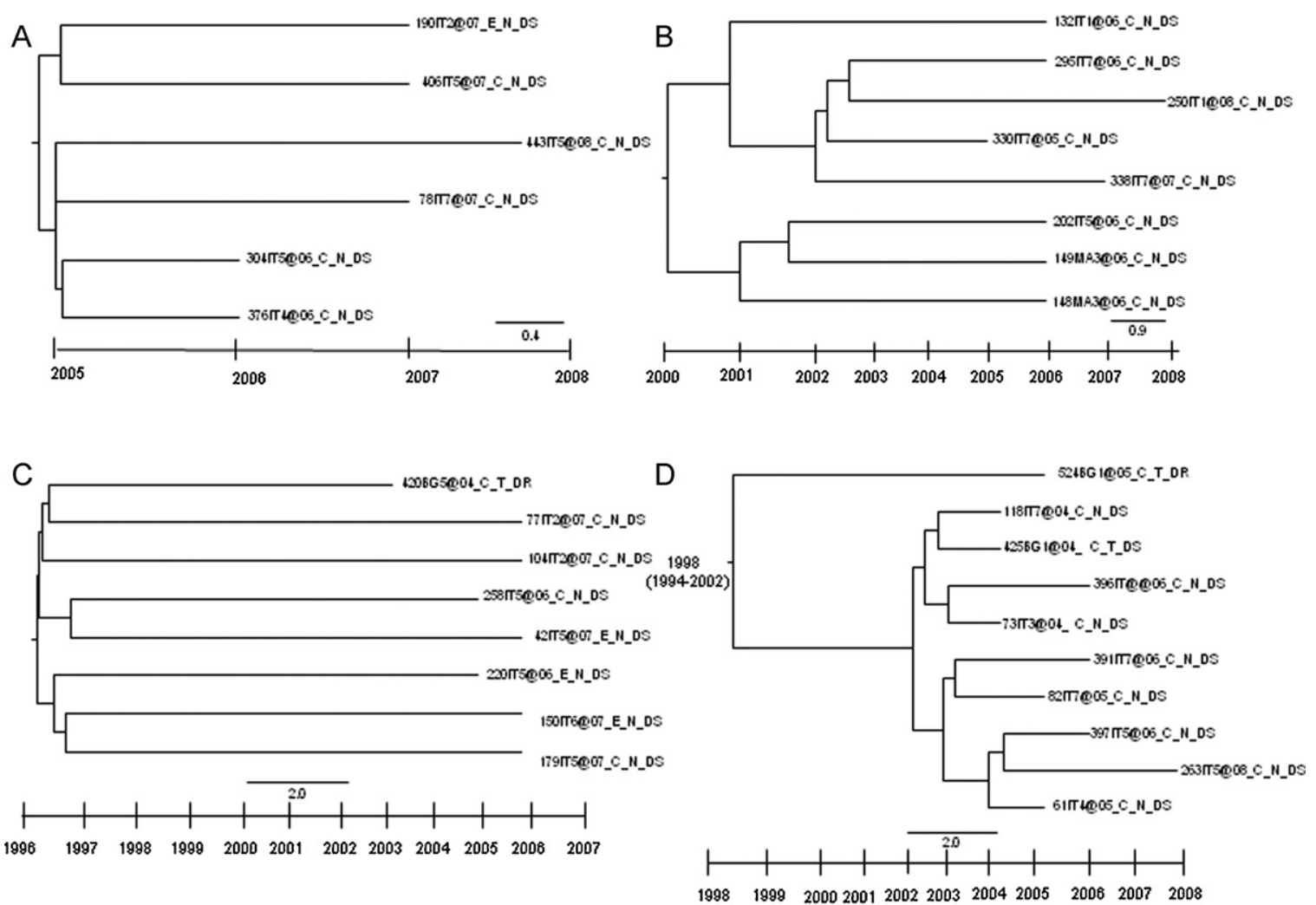

E

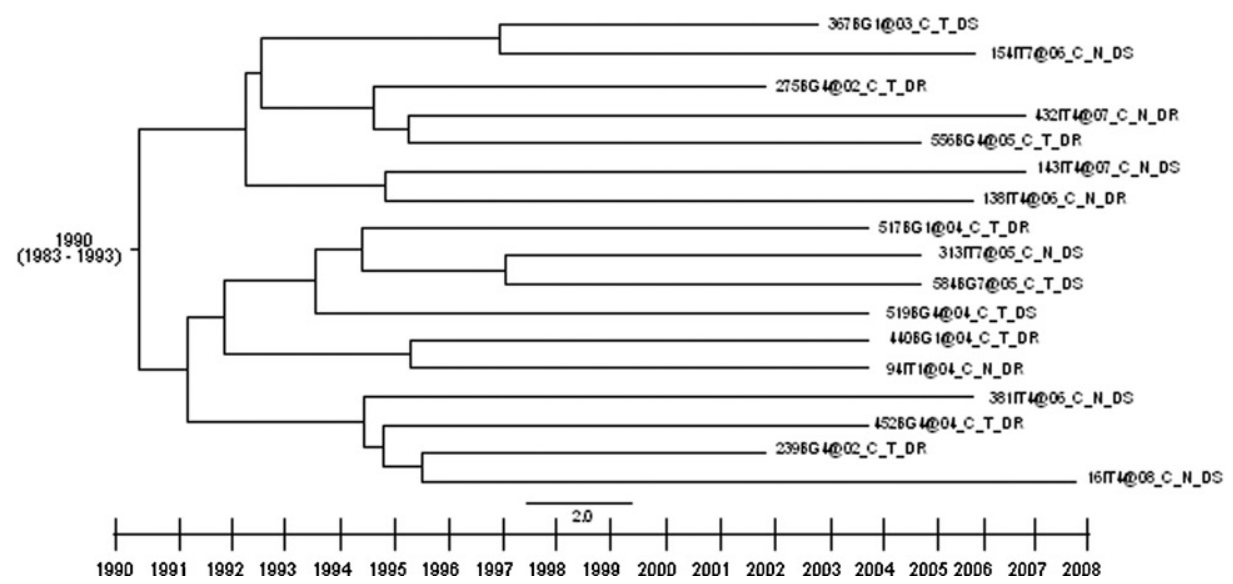

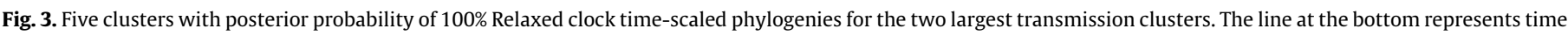

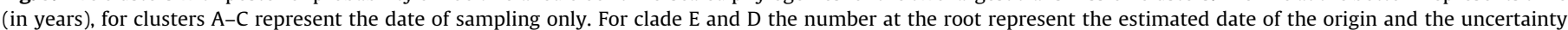

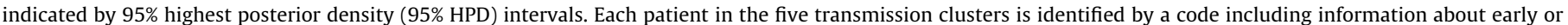
chronical infection (E or $\mathrm{C}$ ), HAART naïve or treated status ( $\mathrm{N}$ or T), drug-resistant or drug-susceptible virus (DR or DS).

of NNRTI-resistance mutations in recent infections compared to chronic infections ( $2.4 \%$ versus $6.1 \%$ ).

A different scenario was observed in chronically infected patients, where $13.3 \%$ carried at least one mutation associated with transmitted drug resistance. In particular, NRTI and NNRTI resistance mutations were found at a frequency of $5.9 \%$ and $5.6 \%$, respectively, while mutations associated with PI resistance only in $1.0 \%$ of patients. Again, these results were consistent with other Italian and European reports (Alteri et al., 2009; Lapadula et al., 2008; UK Collaborative Group on HIV Drug Resistance et al., 2007), even if other European studies have reported a lower prevalence of NNRTI resistance mutations and a higher prevalence of NRTI and PI resistance mutations in their cohort of drug-naïve patients (The SPREAD Programme, 2008; Wensing et al., 2005;
Yerly et al., 2009). These discrepancies among the different studies highlight the need of local analysis, since only such type of studies can provide a proper answer to the prevalence of resistance in drug-naïve patients in that particular setting. Extrapolation of data from other areas, even if contiguous, might bring to erroneous conclusions with potentially relevant clinical consequences.

Approximately $36 \%$ of these drug-resistance strains were involved in significant clusters: $61 \%$ of them clustered with HAART treated patients, while $38 \%$ with only drug-naïve patients. This result, consistent with that shown by Hue et al. (2009) in United Kingdom and Yerly et al. (2009) in the Swiss Cohort, suggests that, beyond treatment-experienced patients, also drug-naïve patients can contribute to the transmission of drug-resistance viruses. In particular, these lineages are indicative of treatment-independent 
Table

\begin{tabular}{|c|c|c|c|c|c|c|c|c|c|c|}
\hline \multirow[t]{2}{*}{ Cluster } & \multirow[t]{2}{*}{ Sequence } & \multirow[t]{2}{*}{ Sampling date } & \multirow[t]{2}{*}{ Risk group } & \multicolumn{3}{|l|}{ Shared drug resistance mutations } & \multirow{2}{*}{$\begin{array}{l}\text { Atypical } \\
\text { mutation }\end{array}$} & \multirow{2}{*}{$\begin{array}{l}\text { Treatment } \\
\text { history }\end{array}$} & \multirow{2}{*}{$\begin{array}{l}\text { Nation } \\
\text { of origin }\end{array}$} & \multirow[t]{2}{*}{ New infection } \\
\hline & & & & NRTI & NNRTI & PI & & & & \\
\hline A & 190IT2.07 & 2007 & Heterosex & - & - & - & & Naïve & Italy & yes \\
\hline A & 406IT5.07 & 2007 & Heterosex & - & - & - & & Naïve & Italy & - \\
\hline A & 443IT5.08 & 2008 & Heterosex & - & - & - & & Naïve & Italy & - \\
\hline A & 78IT7.07 & 2007 & Heterosex & - & - & - & $\mathrm{L}_{210 \mathrm{~S}_{\mathrm{rt}}}$ & Naïve & Italy & - \\
\hline A & 304IT5.06 & 2006 & MSM & - & - & - & & Naïve & Italy & - \\
\hline A & 376IT4.06 & 2006 & Heterosex & - & - & - & & Naïve & Italy & - \\
\hline B & 132IT1.06 & 2006 & MSM & - & - & - & & Naïve & Italy & - \\
\hline B & 295IT7.06 & 2006 & MSM & - & - & - & V82I $I_{\mathrm{pr}}$ & Naïve & Italy & - \\
\hline B & 250IT1.08 & 2008 & Heterosex & - & - & - & & Naïve & Italy & - \\
\hline B & 330IT7.05 & 2005 & MSM & - & - & - & & Naïve & Italy & - \\
\hline B & $338 \mathrm{IT7} 7.07$ & 2007 & MSM & - & - & - & V82I $\mathrm{I}_{\mathrm{pr}}$ & Naïve & Italy & - \\
\hline B & 202IT5.06 & 2006 & Heterosex & - & - & - & $\mathrm{V} 82 \mathrm{I}_{\mathrm{pr}}$ & Naïve & Italy & - \\
\hline B & 148MA3.06 & 2006 & Heterosex & - & - & - & $\mathrm{V} 82 \mathrm{I}_{\mathrm{pr}}$ & Naïve & Marocco & - \\
\hline B & 149MA3.06 & 2006 & Heterosex & - & - & - & & Naïve & Marocco & - \\
\hline C & 420bg5.04 & 2004 & Heterosex & M41L/D67N/T69D/M184V/L210W/T 215Y & - & - & & Treated & Italy & - \\
\hline c & 77IT2.07 & 2007 & MSM & r & - & - & & Naïve & Italy & - \\
\hline c & $104 \mathrm{IT} 2.07$ & 2007 & Heterosex & - & - & - & & Naïve & Italy & - \\
\hline c & 258IT5.06 & 2006 & Heterosex & - & - & - & & Naïve & Italy & - \\
\hline c & 42 IT5.07 & 2007 & Heterosex & - & - & - & & Naïve & Italy & yes \\
\hline c & 150IT6.07 & 2007 & Heterosex & - & - & - & & Naïve & Italy & yes \\
\hline c & 220IT5.06 & 2006 & Heterosex & - & - & - & & Naïve & Italy & yes \\
\hline c & 179IT5.07 & 2007 & MSM & - & - & - & & Naïve & Italy & - \\
\hline D & 524BG1.05 & 2005 & MSM & M184V & K103N/K101E & - & & Treated & Italy & - \\
\hline D & 118IT7.04 & 2004 & MSM & - & - & - & & Naïve & Italy & - \\
\hline D & 425BG1.04 & 2004 & MSM & - & - & - & & Treated & Italy & - \\
\hline D & $396 I T 7.06$ & 2006 & MSM & - & - & - & & Naïve & Italy & - \\
\hline D & 73IT3.04 & 2004 & Heterosex & & & & & Naïve & Italy & - \\
\hline D & 391IT7.06 & 2006 & MSM & - & - & - & $\mathrm{L}_{2} 10 \mathrm{~S}_{\mathrm{rt}}$ & Naïve & Italy & - \\
\hline D & 82 IT7.05 & 2005 & MSM & - & - & - & & Naïve & Italy & - \\
\hline D & 397IT5.06 & 2006 & Heterosex & - & - & - & & Naïve & Italy & - \\
\hline D & 263IT5.08 & 2008 & Heterosex & - & - & - & & Naïve & Italy & - \\
\hline D & 61IT4.05 & 2005 & MSM & - & - & - & & Naïve & Italy & - \\
\hline $\mathrm{E}$ & $367 \mathrm{bg} 1.03$ & 2003 & MSM & - & - & - & V179I $I_{\mathrm{rt}}$ & Treated & Italy & - \\
\hline $\mathrm{E}$ & $154 i t 7.06$ & 2006 & Heterosex & - & - & - & V179I $I_{r t}$ & Naïve & Italy & - \\
\hline E & $275 b g 4.02$ & 2002 & IDU & M184V & K103N & - & - & Treated & Italy & - \\
\hline E & $432 \mathrm{it} 4.07$ & 2007 & Heterosex & M184V & - & - & $\mathrm{V} 179 \mathrm{I}_{\mathrm{rt}}$ & Naïve & Italy & - \\
\hline E & $556 b g 4.05$ & 2005 & Heterosex & M41L/D67N/L210W/T215Y & K103N & - & $\mathrm{K} 101 \mathrm{Q}_{\mathrm{rt}} ; \mathrm{V} 179 \mathrm{I}_{\mathrm{rt}}$ & Treated & Italy & - \\
\hline $\mathrm{E}$ & $143 i t 4.07$ & 2007 & Heterosex & - & - & - & V179I $I_{\mathrm{rt}}$ & Naïve & Italy & - \\
\hline $\mathrm{E}$ & 138it4.06 & 2006 & Heterosex & $\mathrm{M} 41 \mathrm{M} / \mathrm{L}$ & - & - & V179I $I_{\mathrm{rt}}$ & Naïve & Italy & - \\
\hline $\mathrm{E}$ & 517BG1.04 & 2004 & MSM & D67N/K70R/M184V/K219E & - & - & V179I $I_{r t}$ & Treated & Italy & - \\
\hline $\mathrm{E}$ & 313IT7.05 & 2005 & Heterosex & - & - & - & V179I $I_{r t}$ & Naïve & Italy & - \\
\hline $\mathrm{E}$ & 584BG7.05 & 2005 & Heterosex & - & - & - & V179I $I_{r t}$ & Treated & Italy & - \\
\hline $\mathrm{E}$ & 519BG4.04 & 2004 & Heterosex & - & - & - & V179I $I_{\mathrm{rt}}$ & Treated & Italy & - \\
\hline $\mathrm{E}$ & 440BG1.04 & 2004 & MSM & - & G190E & - & $\mathrm{V} 179 \mathrm{I}_{\mathrm{rt}}$ & Treated & Italy & - \\
\hline $\mathrm{E}$ & 94IT1.04 & 2004 & MSM & - & G190E & - & V179I It & Naïve & Italy & - \\
\hline $\mathrm{E}$ & 381IT4.06 & 2006 & Heterosex & - & - & - & - & Naïve & Italy & - \\
\hline $\mathrm{E}$ & 452BG4.04 & 2004 & Heterosex & M41L/D67N/K70R/M184V/L210W/T215F/K219E & G190A & - & ${\mathrm{V} 179 I_{\mathrm{rt}}}_{\mathrm{r}}$ & Treated & Italy & - \\
\hline $\mathrm{E}$ & 239BG4.02 & 2002 & Heterosex & M184V & & $\mathrm{D} 30 \mathrm{~N} / \mathrm{L} 33 \mathrm{~F}$ & V179I $I_{\mathrm{rt}}$ & Treated & Italy & - \\
\hline $\mathrm{E}$ & $16 I T 4.08$ & 2008 & MSM & - & - & - & V106I It; V179I It & Naïve & Italy & - \\
\hline
\end{tabular}


reservoirs of resistance and represent a potential long-term risk to the continued success of antiretroviral therapy.

The treatment-independent transmitted drug-resistance mutations involved in 3 epidemiological events were the TAMs2, D67N and $\mathrm{K} 219 \mathrm{Q}$, the revertant T215D, and the NNRTI resistance mutations K103N. Their presence may be related with the limited effect of these mutations upon viral replicative capacity, and therefore with the increased chances to establish infection in the new host; in particular, the mutations D67N and K103N were known to confer high level of drug resistance without altering the viral replicative capacity (Domaoal et al., 2006), while the mutations K219Q and T215D, frequently found in HIV-1 lineages (Hue et al., 2009), may not significantly change drug-susceptibility on their own and impair viral fitness (Cong et al., 2007; De Ronde et al., 2001). Not surprisingly, PI resistance mutations, observed only in $1 \%$ of our population, were never found in these treatmentindependent transmission networks. The lower prevalence of PI resistance mutations in transmission networks is consistent with data from other cohorts (Brenner et al., 2007, 2008) and could be related with the negative impact on viral replicative fitness of the PI mutations (Martinez-Picado et al., 1999).

Differently, the $61.5 \%$ of drug-naïve patients with transmitted drug-resistance clustered in 6 epidemiological networks along with HAART treated patients. In these clusters, as well as in the 11 clusters involving drug-resistant strains from HAART treated individuals and drug-sensitive strains from drug-naïve individuals, we could frequently observe reversion events of drug-resistance mutations. This could be explained by the substantial loss of HIV-1 genetic diversity during transmission (Keele et al., 2008) and by the fitness cost of the multidrug resistance variants, thus contributing to create a bottleneck for forward transmission of such drugresistant variants (Turner et al., 2004).

An evidence of reversion events could be provided by clade $\mathrm{D}$ and clade E (Fig. 3D and E). It is noteworthy that these clade involved HAART treated patients with multiple resistance mutations, that could be easily lost before transmission to drug-naïve individuals; their absence in the earliest sequences of the cluster could be also suggested by a long transmission interval or transmission through individuals not present in the data set. Differently, the persistence of other drug-resistance mutations as the G190E could be explained by their skill to not alter the viral replication capacity (Domaoal et al., 2006). The persistence of such drug-resistant viruses in drug-naïve patients could also result from short transmission intervals, such that resistance mutations are transmitted to the next individual before reversion occurs.

Two main clades were also characterized by a dated phylogenies reconstruction to provide new insights into the temporal structure of resistance transmission among treated and untreated HIV patients. According to the time-scaled phylogenies, these clusters originated between 1990 (cluster E) and 1997 (cluster D). The 3 drug-resistance lineages originated between 1990 and 1997 (cluster D). The 3 drug-resistance lineages originated between 1990 and 2000, which corresponds to the early years of ART usage and to the time when the transmission of drug-resistance mutations was at its highest level in Europe and in United States (Grant et al., 2002). Interestingly, no dated clusters involving only drug-susceptible strains sampled, between 2005 and 2008 (A e B), can support the reduction of transmitted drug-resistance in drugnaïve patients recently highlighted in Europe (Alteri et al., 2009; The SPREAD Programme, 2008; UK Collaborative Group on HIV Drug Resistance et al., 2007; Yerly et al., 2007).

In conclusion, our findings highlight the role of newly diagnosed individuals, not yet exposed to antiretroviral drugs, in the transmission of drug-resistant HIV-1 strains, despite the reduction of drug-resistance in new infections. Overall, these findings provide new insights for the planning and management of treatment programs in developing countries.

\section{Acknowledgements}

These data were a part of the training of one of the authors (A.C.) at the 15th International Bioinformatics Workshop on Virus Evolution and Molecular Epidemiology, Rotterdam, The Nederlands, 7th-11th September 2009, and were partially presented there. http://www.rega.kuleuven.be/cev/workshop. This work was financially supported by grants from CHAIN, Collaborative HIV and Anti-HIV Drug-Resistance Network, Integrated Project no. 223131, funded by the EuropeanCommission Framework-7 Program, and from the Italian Ministry of Health (CUP: E81J10000000001).

\section{Appendix A. Supplementary data}

Supplementary data associated with this article can be found, in the online version, at doi:10.1016/j.meegid.2011.01.019.

\section{References}

Alteri, C., Svicher, V., Gori, C., D’Arrigo, R., Ciccozzi, M., Ceccherini-Silberstein, F., Selleri, M., Bardacci, S.A., Giuliani, M., Elia, P., Scognamiglio, P., Orchi, N., Giradi, E., Perno, C.F., SENDIH Study Group, 2009. Characterization of the pattern of drug-resistance mutations in newly diagnosed HIV-1 infected patients naïve to the antiretroviral drugs. BMC Infect. Dis. 16, 9-111.

Baldanti, F., Paolucci, S., Ravasi, G., Maccabruni, A., Moriggia, A., Barbarini, G., Maserati, R., 2008. Changes in circulation of B and non-B HIV strains: spotlight on a reference centre for infectious disease in Northern Italy. J. Med. Virol. 80, 947-952.

Balotta, C., Facchi, G., Violin, M., van Dooren, S., Cozzi-Lepri, A., Forbici, F., Bertoli, A., Riva, C., Senese, D., Caramello, P., Carnevale, G., Rizzardini, G., Cremonini, L., Monno, L., Rezza, G., Perno, C.F., Ippolito, G., d'Arminio-Monforte, A., Vandamme, A.M., Moroni, M., ICONA Study Group, 2001. Increasing prevalence of non-clade B HIV-1 strains men and women as monitored by analysis of reverse transcriptase and protease sequences. J. AIDS 27, 499-505.

Bennett, D.E., Camacho, R.J., Otelea, D., Kurizkes, D.R., Fleury, H., Kiuchi, M., Heneine, W., Kantor, R., Schapiro, J.M., Vandamme, A.M., Sandstrom, P., Boucher, C.A., van de Vijver, D., Rhee, S.Y., Liu, T.F., Pillay, D., Shafer, R.W., 2009. Drug resistance mutations for surveillance of transmitted HIV-1 drug-resistance: 2009 update. PLoS One 4, 1-8.

Brenner, B.G., Roger, M., Moisi, D.D., Oliveira, M., Hardy, I., Turgel, R., Charest, H., Routy, J.P., Wainberg, M.A., Montreal PHI Cohort, HIV Prevention Study Group, 2008. Transmission networks of drug resistance acquired in primary/early stage HIV infection. AIDS 22, 2509-2515.

Brenner, B.G., Roger, M., Routy, J.P., Moisi, D., Ntemgwa, M., Matte, C., Baril, J.C., Thomas, R., Rouleau, D., Bruneau, J., Leblanc, R., Legault, M., Tremblay, C., Charest, H., Waimberg, M.A., Quebec Primary HIV Infection Study Group, 2007. High rates of forward transmission events after acute/early HIV-1 infection. J. Infect. Dis. 195, 951-959.

Buonaguro, L., Petrizzo, A., Tagliamonte, M., Vitone, F., Re, M.C., Pilotti, E., Casoli, C., Sbreglia, C., Perrella, O., Tornesello, M.L., Buonaguro, F.M., 2008. Molecular and phylogenetic analysis of HIV-1 variants circulating in Italy. Infect. Agents Cancer 10, 3-13.

Castelnuovo, B., Chiesa, E., Rusconi, S., Adorni, F., Bongiovanni, M., Melzi, S., Cicconi, P., Tordato, F., Meroni, L., Bini, T., d'Arminio-Monforte, A., 2003. Declining incidence of AIDS and increasing prevalence of AIDS presenters among AIDS patients in Italy. Eur. J. Clin. Microbiol. Infect. Dis. 22, 663-669.

Ciccozzi, M., Montieri, S., Salemi, M., De Oliveira, T., Dorrucci, M., Sinicco, A., De Luca, A., Giuliani, M., Balotta, C., Rezza, G., 2007. An outbreak of HIV-1 subtype G among Italian injecting drug users. AIDS 21, 1213-1215.

Cong, M.E., Heneine, W., Garcia-Lerma, J.C., 2007. The fitness cost of mutations associated with human immunodeficiency virus type 1 drug resistance is modulated by mutational interactions. J. Virol. 81, 3037-3041.

Costagliola, D., Descamps, D., Assoumou, L., Morand-Joubert, L., Marcelin, A.G., Brodard, V., Delaugerre, C., Mackiewicz, V., Ruffault, A., Izopet, J., Plantier, J.C., Tamalet, C., Yerly, S., Saidi, S., Brun-Vezinet, S., Masquelier, B., Agence Nationale de Recherches sur le SIDA et les Hepatites Virales (ANRS) AC11 Resistance Study Group, 2007. Prevalence of HIV- drug resistance in treated patients: a French nationwide study. J AIDS 46, 12-28.

De Ronde, A., van Dooren, M., van Der Hoek, L., Bouwhuis, D., de Rooij, E., van Gemen, B., de Boer, R., Goudsmit, J., 2001. Establishment of new transmissible and drug-sensitive human immunodeficiency virus type 1 wild types due to transmission of nucleoside analogue-resistant virus. J Virol. 75, 595-602. 
Domaoal, R.A., Bambara, R.A., Demeter, L.M., 2006. HIV-1 reverse transcriptase mutants resistant to non-nucleoside reverse transcriptase inhibitors do not adversely affect DNA synthesis: pre-steady-state and steady-state kinetic studies. J. AIDS 42, 405-411.

Drummond, A.J., Rambaut, A., 2007. BEAST: Bayesian evolutionary analysis by sampling trees. BMC Evol. Biol. 7, 214.

Grant, R.M., Hecht, F.M., Warmerdam, M., Liu, L., Liegler, T., Petropoulos, C.J., Hellmann, N.S., Chesney, M., Busch, M.P., Kahn, J.O., 2002. Time trends in primary HIV-1 drug resistance among recently infected persons. JAMA 288, $181-188$.

Hall, T.A., 1999. Bioedit: a user-friendly biological sequence alignment editor and analysis program for Windows 95/98 NT. Nucleic Acids Symp. Ser. 41, 95-98.

Hue, S., Gifford, R.J., Dunn, D., Fernhill, E., Pillay, D., UK Collaborative Group on HIV Drug Resistance, 2009. Demonstration of sustained drug-resistant human immunodeficiency virus type 1 lineages circulating among treatment-naïve individuals. J. Virol. 83, 2645-2654.

Huelsenbeck, J.P., Ronquist, F., 2001. MRBAYES: Bayesian inference of phylogenetic trees. Bioinformatics 17 (8), 754-755

Kass, R.E., Raftery, A.E., 1995. Bayes factors. J. Am. Stat. Assoc. 90, 773-795.

Keele, B.F., Giorgi, E.E., Salazar-Gonzalez, J.F., 2008. Identification and characterization of transmitted and early founder virus envelopes in primary HIV-1 infection. Proc. Natl. Acad. Sci. U.S.A. 105, 7552-7557.

Lapadula, G., Izzo, I., Gargiulo, F., Paraninfo, G., Castelnuovo, F., Quiros-Roldan, E., Cologni, G., Ceresoli, F., Manca, N., Carosi, G., Torti, G., 2008. Updated prevalence of genotypic resistance among HIV-1 positive patients naïve to antiretroviral therapy: a single center analysis. J. Med. Virol. 80, 747-753.

Martinez-Picado, J., Savara, A.V., Sutton, L., D’Aquila, R.T., 1999. Replicative fitness of protease inhibitor-resistant mutants of human immunodeficiency virus type 1 . J. Virol. 73, 3744-3752.

Pillay, D., Taylor, S., Richman, D.D., 2000. Incidence and impact of resistance against approved antiretroviral drugs. Rev. Med. Virol. 10, 231-253.

Schmidt, H.A., Strimmer, K., Vingron, M., von Haeseler, A., 2002. TREE-PUZZLE: maximum likelihood phylogenetic analysis using quartets and parallel computing. Bioinformatics 18, 502-504.

Suligoi, B., Boros, S., Camoni, L., Lepore, D., 2009. Aggiornamento delle nuove diagnosi di infezione da HIV al 31 dicembre 2007 e dei casi di AIDS in Italia al 31 dicembre 2008. Notiziario dell' Istituto Superiore di Sanità 22 (1), 3-27.

Suchard, M.A., Weiss, R.E., Sinsheimer, J.S., 2001. Bayesian selection of continuoustime Markov chain evolutionary models. Mol. Biol. Evol. 18, 1001-1013.

The SPREAD Programme, 2008. Transmission of drug-resistant HIV-1 in Europe remains limited to single classes. AIDS 22, 625-635.

Thompson, J.D., Gibson, T.J., Plewniak, F., Jeanmougin, F., Higgins, D.G., 1994. CLUSTAL_X windows interface: flexible strategies for multiple sequence alignment aided by quality analysis tools. Nucleic Acids Symp. Ser. 25, 4876-4882.

Tozzi, V., Zaccarelli, M., Bonfigli, S., Lorenzini, P., Liuzzi, G., Trotta, M.P., Forbici, F., Gori, C., Bertoli, A., Bellagamba, R., Narciso, P., Perno, C.F., Antinori, A., Collabo- rative Group for Clinical Use of HIV Genotype Resistance Test, 2006. Drug-classwide resistance to antiretrovirals in HIV-infected patients failing therapy: prevalence, risk factors and virological outcome. Antivir. Ther. 11, 553-560.

Turner, D., Brenner, B., Routy, J.P., Moisi, D., Rosberger, Z., Roger, M., Wainberg, M.A., 2004. Diminished representation of HIV-1 variants containing select drug resistance-conferring mutations in reverse transcriptase in primary HIV infection. J. AIDS 37, 1627-1631.

UK Collaborative Group on HIV Drug Resistance, UK Collaborative HIV Cohort Study, UK Register of HIV Seroconverters, 2007. Evidence of a decline in transmitted HIV-1 drug-resistance in the United Kingdom. AIDS 21, 1035-1039.

Violin, M., Cozzi-Lepri, A., Velleca, R., Vincenti, A., D’Elia, S., Chiodo, F., Ghinelli, F., Bertoli, A., d'Arminio Monforte, A., Perno, C.F., Moroni, M., Balotta, C., 2004a. Risk of failure in patients with 215 HIV-1 revertants starting their first thymidine analog-containing highly active antiretroviral therapy. AIDS 18 , 227-235.

Violin, M. Velleca, R, Cozzi-Lepri, A, Riva, C. Grossi, P.A., Carnevale, g., Rizzardini, G., Petrelli, E., Perno, C.F., Monforte, A., Balotta, C., ICONA Study Group, 2004b. Prevalence of HIV-1 Primary Drug Resistance in Seroconverters of the ICoNA Cohort Over the Period 1996-2001. J. AIDS 36, 761-764.

Walker, P.R., Pybus, O.G., Rambaut, A., Holmes, E.C., 2005. Comparative population dynamics of HIV-1 subtypes B and C: subtype-specific differences in patterns of epidemic growth. Infect. Genet. Evol. 5, 199-208.

Wensing, A.M., van de Vijver, D.A., Angarano, G., Asjo, B., Balotta, C., Boeri, E., Camacho, R., Chaix, M.L., Costagliola, D., De Luca, A., Derdelinckx, I., Grossman, Z., Hamouda, O., Hatzakis, A., Hemmer, R., Hoepelman, A., Horban, A., Korn, K., Kucherer, C., Leitner, T., Loveday, C., MacRae, E., Malikovic, I., de Mendoza, C., Meyer, L., Nielsen, C., Op de Coul, E.L., Ormaasen, V., Paraskevis, D., Perrin, L., Puchhammer-Stockl, E., Ruiz, L., Salminen, M., Schmit, J.C., Schneider, F., Schuurman, R., Soriano, V., Stanczak, G., Stanojevic, M., Vandamme, A.M., Van Laethem, K., Violin, M., Yerly, S., Zazzi, M., Boucher, C.A 2005. SPREAD Programme Prevalence of drug-resistant HIV-1 variants in untreated individuals in Europe: implications for clinical management. J. Infect. Dis. 192, 958-966.

Yerly, S., Junier, T., Gayet-Ageron, A., Amari, E.B., von Wyl, V., Günthard, H.F. Hirschel, B., Zdobnov, E., Kaiser, L., 2009. Swiss HIV Cohort Study The impact of transmission clusters on primary drug resistance in newly diagnosed HIV-1 infection. AIDS 23, 1415-1423.

Yerly, S., von Wyl, V., Ledergerber, B., Boni, J., Schupbach, J., Burgisser, P., Klimkait, T., Rickenbach, M., Kaiser, L., Gunthard, H.F., Perrin, L., Swiss HIV Cohort Study, 2007. Transmission of HIV-1 drug-resistance in Switzerland: a 10-year molecular epidemiology survey. Antivir. Ther. 21, 2223-2229.

Yerly, S., Vora, S., Rizzardi, P., Chave, J.P., Vernazza, P.L., Flepp, M., Telenti, A., Battegay, M., Veuthey, A.L., Bru, J.P., Rickenbach, M., Hirschel, B., Perrin, L. Swiss HIV Cohort Study, 2001. Acute HIV infection: impact on the spread of HIV and transmission of drug resistance. AIDS 15 (17 (November)), 2287-2292. 